\title{
Overdiagnosis of high-grade dysplasia in Barrett's esophagus: a multicenter, international study
}

\author{
Nikhil A Sangle ${ }^{1}$, Shari L Taylor ${ }^{2}$, Mary J Emond ${ }^{3}$, Michelle Depot ${ }^{4}$, Bergein F Overholt ${ }^{5}$, \\ Mary P Bronner ${ }^{1,6}$ and On behalf of the International Photodynamic Group for High-Grade \\ Dysplasia in Barrett's Esophagus
}

\begin{abstract}
${ }^{1}$ Department of Pathology, Schulich School of Medicine and London Health Sciences Centre, London, Ontario, Canada; ${ }^{2}$ Miraca Life Sciences, Dallas, TX, USA; ${ }^{3}$ Department of Biostatistics, University of Washington, Seattle, WA, USA; ${ }^{4}$ Pinnacle Biologics, Bannockburn, IL, USA; ${ }^{5}$ Thompson Cancer Survival Center, Knoxville, TN, USA and ${ }^{6}$ Department of Pathology, University of Utah and ARUP Laboratories, Salt Lake City, UT, USA
\end{abstract}

\begin{abstract}
Numerous histological mimics of high-grade dysplasia in Barrett's esophagus predispose to overdiagnosis and potential serious mismanagement, including unnecessary esophagectomy. This study investigates the prevalence and sources of this problem. Biopsies from 485 patients diagnosed with Barrett's high-grade dysplasia were screened for a multi-institutional, international Barrett's endoscopic ablation trial. Screening included review of the original diagnostic slides and an additional protocol endoscopy with an extensive biopsy sampling. Observer variability by the study pathologists was assessed through two blinded diagnostic rounds on 437 biopsies from 26 random study endoscopies. Study diagnostic reassessments revealed significantly lower rates of high-grade dysplasia. Only 248 patients $(51 \%)$ were confirmed to have high-grade dysplasia. The remaining patients had inflamed gastric cardia without Barrett's $(n=18 ; 7 \%)$, Barrett's without dysplasia $(n=35$; $15 \%)$, indefinite change $(n=61 ; 26 \%)$, low-grade dysplasia $(n=79 ; 33 \%)$, adenocarcinoma $(n=43 ; 18 \%)$, and other $(n=1 ;<1 \%)$, yielding an alarming total of 194 or $40 \%$ of patients who were overdiagnosed with Barrett's highgrade dysplasia. Study pathologists achieved a high-level agreement $(90 \%$ three-way inter-observer agreement per biopsy, Kappa value 0.77 ) for high-grade dysplasia. Confounding factors promoting overdiagnosis included Barrett's inflammatory atypia $(n=182)$, atypia limited to the basal metaplastic glands $(n=147)$, imprecise criteria for low grade neoplasia $(n=102)$, tangential sectioning artifact $(n=59)$, and reactive gastric cardiac mucosa $(n=38)$. A total of 194 patients $(40 \%)$ were overdiagnosed with Barrett's high-grade dysplasia, as affirmed by the extensive screening process and high-level study pathologist agreement. The multiple diagnostic pitfalls uncovered should help raise pathologists' awareness of this problem and improve diagnostic accuracy. Modern Pathology (2015) 28, 758-765; doi:10.1038/modpathol.2015.2; published online 13 February 2015
\end{abstract}

The pathological and clinical criteria, as well as the management of Barrett's esophagus have undergone a considerable change since the condition was first described in 1957 by the influential British surgeon, Dr Norman Barrett. ${ }^{1}$ Neoplastic change, including both dysplasia and carcinoma in Barrett's esophagus is prone to overdiagnosis because of the prominent histological overlap between metaplastic, inflamma-

Correspondence: Dr MP Bronner, University of Utah/ARUP Laboratories, Department of Pathology, Huntsman Cancer Institute, Room N3100, 1950 Circle of Hope, Salt Lake City, UT 84112, USA.

E-mail: mary.bronner@hsc.utah.edu

Received 18 June 2014; revised 17 November 2014; accepted 3 December 2014; published online 13 February 2015 tory, regenerative, and neoplastic pathology in this challenging condition. Overdiagnosis of high-grade dysplasia is especially unfortunate considering its aggressive management consequences, including esophagectomy or endoscopic treatment modalities. ${ }^{2}$

The magnitude of the overdiagnosis problem has not been systematically evaluated. We sought to examine the prevalence and sources of this problem, based on patients undergoing screening for an endoscopic ablation trial of Barrett's esophagus with high-grade dysplasia. ${ }^{3,4}$ To help determine the validity of the trial diagnoses, the three study pathologists (SLT, MPB and late Dr Rodger C Haggitt) were evaluated for observer variability on a per endoscopy and per biopsy basis. The patients also underwent an additional protocol screening 
endoscopy with intensive biopsy sampling (fourquadrant jumbo biopsies every $2 \mathrm{~cm}$ throughout the Barrett's segment with additional biopsies of any visible lesions). Finally, the diagnostic pitfalls leading to overdiagnosis of Barrett's high-grade dysplasia were cataloged from this series to explain the sources of error and increase pathologists' awareness in this difficult area of diagnostic pathology.

\section{Materials and methods}

\section{Subject Selection}

Following institutional review board approval, esophageal biopsies from 485 patients carrying a local institutional diagnosis of Barrett's esophagus with high-grade dysplasia were reviewed for patient inclusion into an endoscopic ablation trial with long-term follow-up proceeding from 1999 through 2005. ${ }^{3,4}$ Barrett's esophagus was defined according to criteria of the American College of Gastroenterology, whereby endoscopic glandular (pink) mucosa within the anatomic tubular esophagus, as well as histological intestinal metaplasia with goblets cells on biopsy histology, were both required. ${ }^{2}$

Patients were enrolled from both academic and private centers at 25 study sites from throughout USA, two sites from Canada, two sites from the UK, and one site from France. The original diagnostic slides were reviewed and a repeat protocol endoscopy was performed by an experienced study gastroenterologist on 485 patients presenting for screening, obtaining four-quadrant jumbo biopsies every $2 \mathrm{~cm}$ throughout the Barrett's segment and additional biopsies of any visible lesion(s).

\section{Histology}

Three experienced gastrointestinal pathologists practicing in a high-volume Barrett's setting (late Dr Rodger C Haggitt, MPB, and SLT), shared the review of all of the pathology for the trial. All biopsies were processed by a single central histology laboratory, where they were routinely fixed in $10 \%$ neutral buffered formalin, processed, paraffinembedded, step and serial sectioned, and stained by hematoxylin and eosin. Consensus neoplasia grading criteria were used. ${ }^{5}$ Specifically, high-grade dysplasia was characterized by a combination of architectural and cytological abnormalities, although cytological alterations were typically most heavily weighted. Cytologically, high-grade dysplasia variably exhibited greater degrees of nucleomegaly, irregularity of nuclear membranes, hyperchromasia, and/or pleomorphism than lower grade diagnoses. However, because these criteria represent continuous variables for which precisely defined cutoffs are not possible, they were considered more subjective and weighted less heavily. Loss of nuclear polarity, as a more dichotomous and therefore more objective criterion, was weighted most heavily in the diagnosis of highgrade Barrett's dysplasia. Loss of nuclear polarity was defined as loss of the orderly parallel orientation of nuclei, and loss of the perpendicular relationship between the nuclei and the basal lamina. High-grade nuclei also tended to become more irregular with the loss of nuclear polarity, in comparison with the pencillate, elongated nuclei of intestinalized lowgrade dysplasia. Architectural alterations of highgrade dysplasia variably included gland crowding, cribriform gland patterns, asymmetrical gland shapes, and/or villiform mucosal configurations. Necrotic or apoptotic debris in dilated glandular lumina was considered highly abnormal and indicative of at least high-grade dysplasia. ${ }^{5-8}$

\section{Observer Variability}

Pre-ablation treatment and post-ablation treatment biopsies were used to permit sufficient observer variability study sample size in combination with proper timing of pathologist readings to avoid calendar bias, expectation bias, and recall bias. Endoscopic procedures were selected for the observer variability portion of this study according to a randomization protocol that was initiated after post-ablation treatment, as per endoscopy biopsy sets became available, and ensuring that both preand post-ablation treatment biopsy sets for this study would be evaluated for observer variability during the same week. This strategy avoids calendar bias in the results. For each post-treatment biopsy set selected, a pre-treatment biopsy set with matching sites was also selected, limiting the number of per endoscopy biopsy sets from any one endoscopy center to no more than three. Thirteen pre- and thirteen post-treatment biopsy sets, all obtained by study endoscopists and prepared at the reference pathology center, were thus sampled. All three pathologists evaluated each biopsy set per endoscopy twice (rounds 1 and 2) to assess for both interand intra-observer variability. The pathologists were blinded to all clinical data, including patient name and demographics, study site, and pre- or postendoscopic ablation treatment status. In addition, to avoid expectation bias, the biopsy sets per endoscopy for the observer variability study were inserted blindly into the stream of cases read daily for the clinical trial, so that at the time of microscopy, the pathologists had no knowledge of whether a case was part of the observer variability study, the main study, round 1 , or round 2 . The interval between the first and second readings by a specific pathologist for any given biopsy set was strictly maintained to be at least 4 weeks, in order to minimize case recall. The sample size of 26 was chosen to provide a confidence interval of $\pm 10 \%$ for estimates of agreement (see below). The selection, order and timing of all study ratings for each pathologist were overseen by the study statistician 
(MJE), who met with laboratory personnel on a weekly basis or more. Each individual biopsy per paraffin block was evaluated separately (about four biopsies per block), and overall grades per block and per endoscopy were determined by the most advanced neoplastic histology.

\section{Statistical Analysis of Observer Variability}

The primary analysis was to estimate agreement for the overall histological diagnosis on a per biopsy basis, since this is the most stringent analysis of pathologist agreement and therefore most relevant to the accuracy of the study pathologists' interpretations. Agreement on the following outcomes was analyzed: (1) high-grade dysplasia, (2) adenocarcinoma, (3) high-grade dysplasia or adenocarcinoma, (4) dysplasia (low grade or high grade), and (5) Barrett's esophagus. Inter-observer agreement was estimated on a pair-wise basis for the three pairs of pathologists, and the three pair-wise estimates for each outcome pooled for an overall estimate of agreement. Two raters were said to agree on a particular outcome (high-grade dysplasia, for example) if both pathologists in the pair diagnosed highgrade dysplasia. Likewise, intra-observer agreement was estimated for each of the three pathologists for each outcome, and the three estimates pooled for an overall estimate. The regression method of 'Generalized Estimating Equations' with Wald-type confidence intervals was used to obtain the pooled ('marginal') estimates ${ }^{9}$ while adjusting for covariates such as presence of inflammation. Kappa statistics were calculated as a second measure of agreement. ${ }^{10}$ Regression analysis was used to evaluate factors affecting agreement, including the presence of obscuring active inflammation/erosions/ulcerations, pre- or post-treatment status; pathologist assignment that high-grade dysplasia could not be excluded, round 1 versus 2 assessment, and the number of biopsies per endoscopy. The above analyses were repeated for agreement by endoscopy as well as by biopsy.

\section{Results}

\section{Screening Endoscopy Sampling}

Using the four-quadrant biopsy protocol every $2 \mathrm{~cm}$ throughout the patients' Barrett's segments, an average of 20 biopsies were obtained per patient per screening endoscopy.

\section{Comparison between Submitting and Study Diagnosis}

Of the 485 screened patients, only 248 (51\%) had confirmed high-grade dysplasia by the study pathologists. In 194 patients (40\%), high-grade dysplasia was reinterpreted as lower grade or absent dysplasia or no Barrett's at all, but rather inflamed cardia
Table 1 Reinterpreted diagnoses in 485 patients originally diagnosed with Barrett's high-grade dysplasia.

\begin{tabular}{lcc}
\hline $\begin{array}{l}\text { Reinterpreted diagnoses from high-grade } \\
\text { dysplasia }\end{array}$ & $\begin{array}{c}\# \\
\text { Patients }\end{array}$ & $\begin{array}{c}\text { Percentages } \\
(\%)\end{array}$ \\
\hline $\begin{array}{l}\text { Reactive gastric cardia mucosa only } \\
\text { Barrett's esophagus, negative for dysplasia }\end{array}$ & 18 & 7 \\
Barrett's esophagus, indefinite for dysplasia & 35 & 15 \\
Barrett's esophagus, low-grade dysplasia & 79 & 26 \\
Other (squamous neoplasia) & 1 & 33 \\
Barrett's adenocarcinoma & 43 & 1 \\
Total & 237 & 18 \\
\hline
\end{tabular}
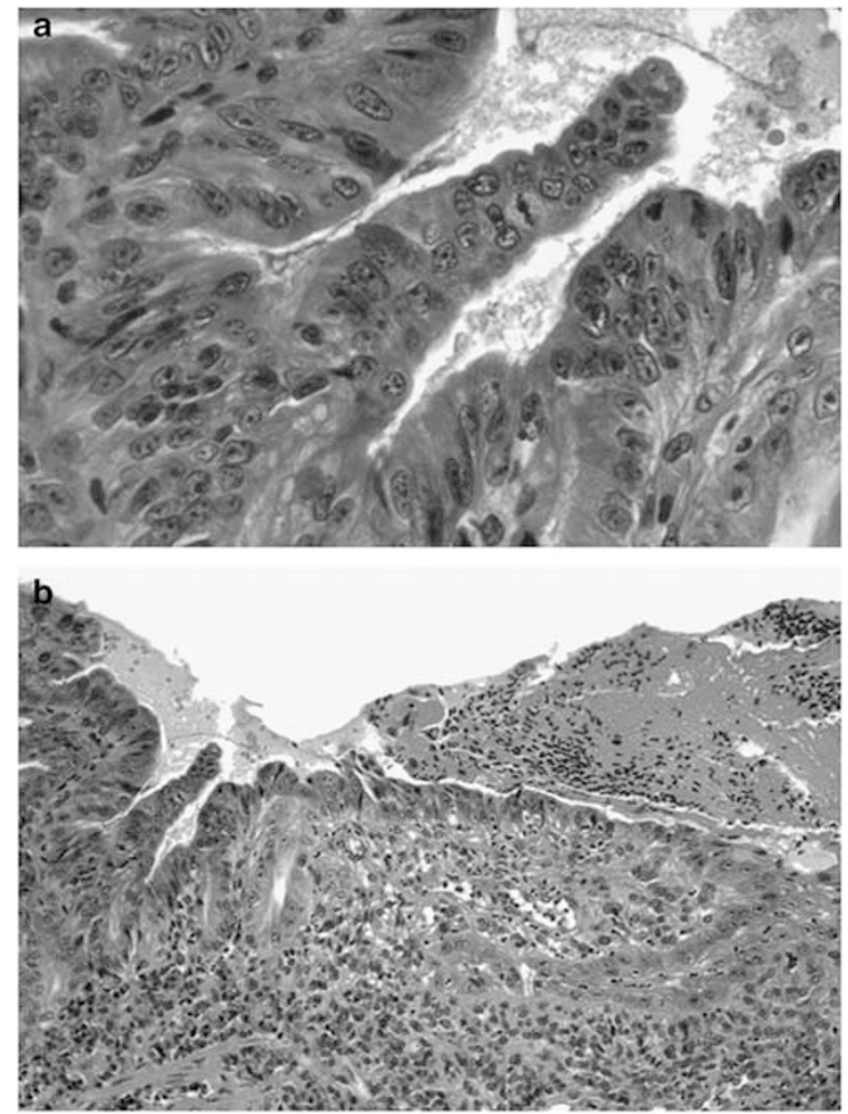

Figure 1 Cytological atypia in moderately to severely inflamed biopsies can readily mimic high-grade dysplasia in Barrett's esophagus (a and $\mathbf{b}$ ). Higher magnification illustrates epithelium mimicking all of the cytological features of high-grade dysplasia (a) directly adjacent to an ulcer (b), indicating that is probably reactive. Diagnostic caution is essential in the setting of active inflammation, especially with ulceration/erosion. A diagnosis of indefinite for dysplasia is appropriate in these settings. Additional commentary that high-grade dysplasia or even carcinoma cannot be excluded may also be warranted with severe atypia as seen in this example.

(Table 1). The remaining 43 patients (9\%) were determined to have Barrett's adenocarcinoma.

The factors leading to overdiagnosis were inflammatory atypia in Barrett's mucosa $(n=182)$ (Figure 1$)$; baseline deep metaplastic glandular atypia with 

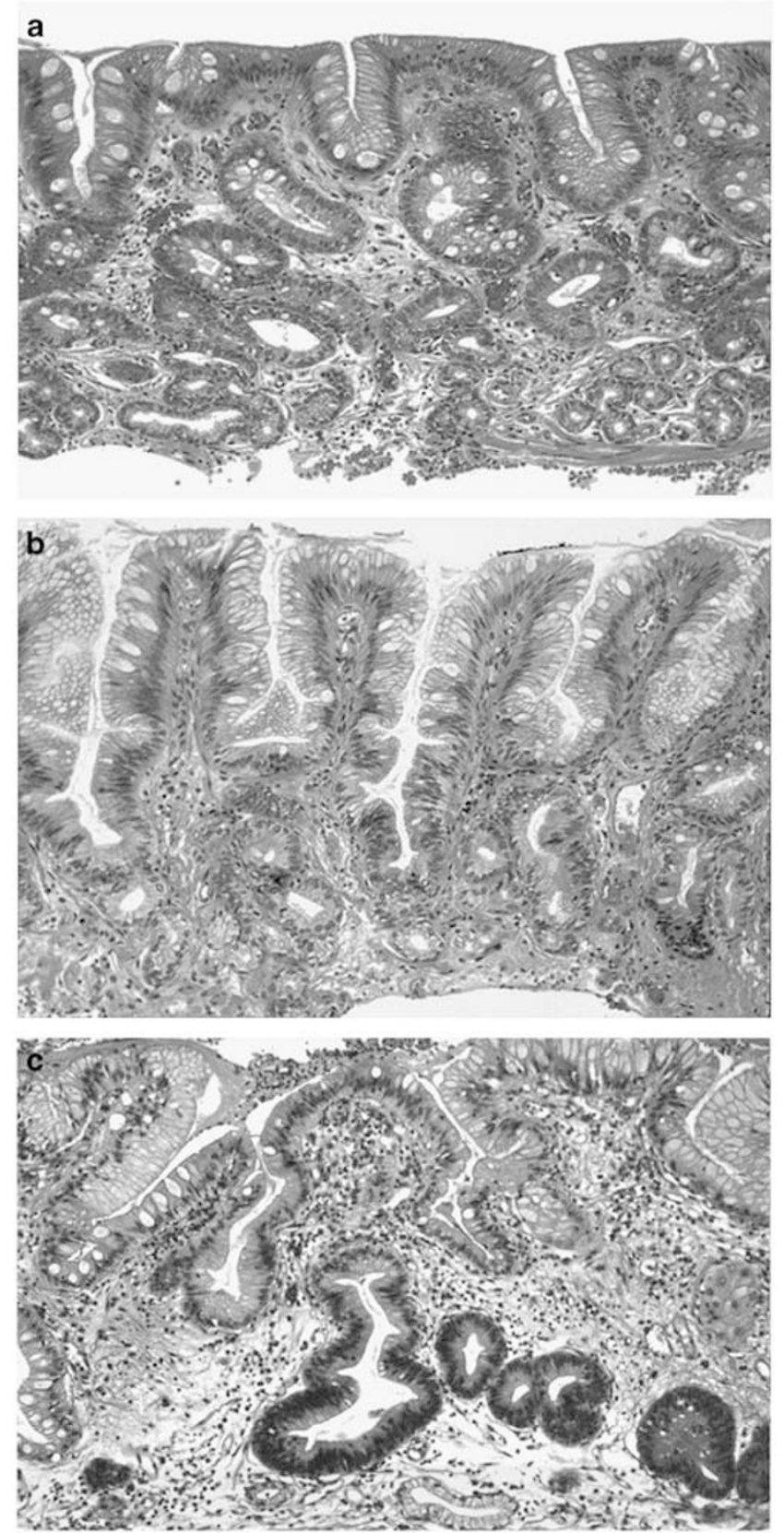

Figure 2 Baseline nondysplastic atypia of Barrett's metaplasia commonly mimics dysplasia. Its nondysplastic nature is revealed by the fact that the atypia is limited to the regenerative compartment at the base of the mucosa with normal maturation to normal nuclei as the epithelium extends onto the mucosal surface (a-c). Metaplastic basal glandular atypia exhibits a spectrum from minimal (a) to intermediate (b) to striking (c). The deep crypts show many or all of the features of dysplasia but surface maturation excludes dysplasia.

surface maturation $(n=147)$ (Figure 2); tangential sectioning artifact $(n=59)$ (Figure 3$)$; inflamed reactive gastric cardia without Barrett's $(n=38)$ (Figure 4); and imprecise criteria for distinguishing high-grade dysplasia from lower grades of neoplasia $(n=102)$ (Figure 5).

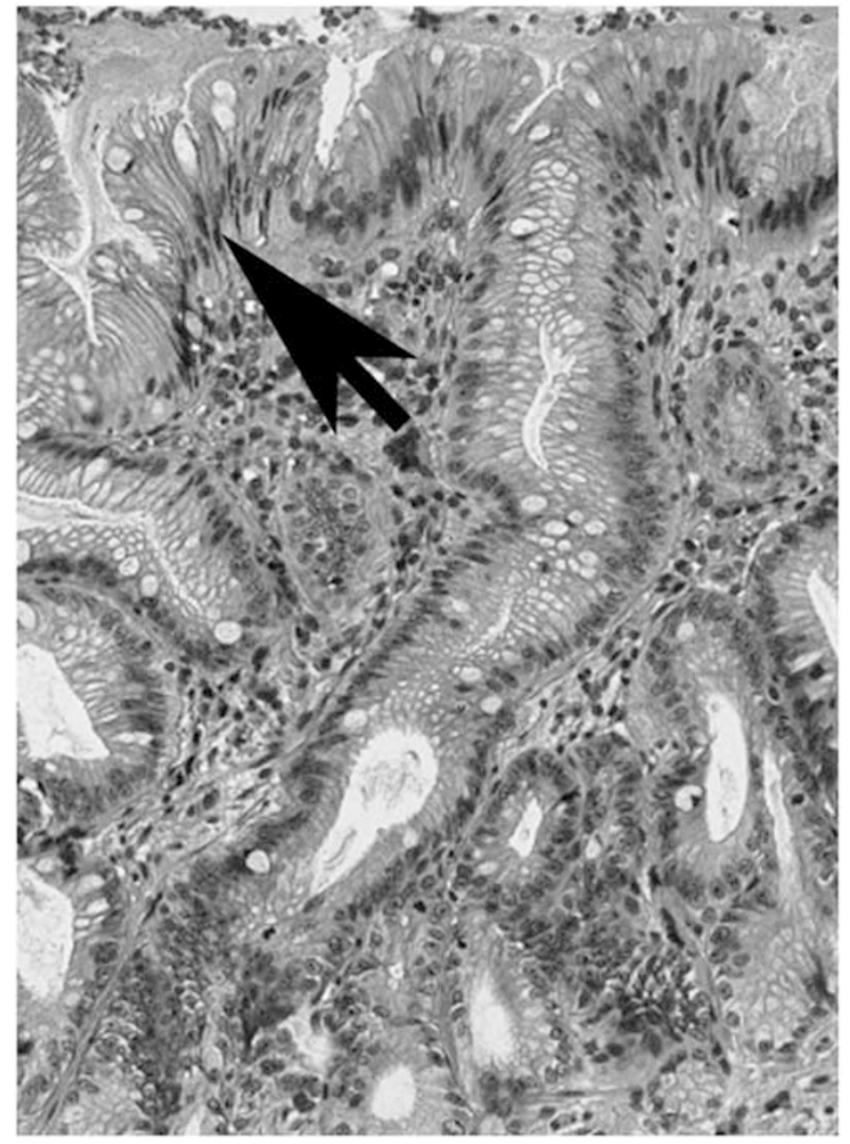

Figure 3 Tangential sectioning artifact creates the appearance of nuclear stratification that may also mimic dysplasia. Two helpful features to avoid overdiagnosing tangential sectioning are first that the cytoplasm is also elongated in addition to the nuclei of the same surface cells (arrow) with tangential sectioning. Second, nuclear stratification as the sole criterion for dysplasia should be used with great caution, if ever.

\section{Observer Variability: Study Composition}

Twenty-six endoscopic procedures were randomly selected from the screening phase of the trial (preablation treatment, $n=13$ ) and the trial phase (post treatment, $n=13$ ). This resulted in 437 total biopsies for evaluation of observer variability. The median number of biopsies per endoscopy was 16.5 (range 2-32). The 13 screening phase endoscopy sets included the following diagnoses (determined by consensus after the study): 0 adenocarcinoma; 7 high-grade dysplasias; 3 low-grade dysplasias; 0 indefinite for dysplasia; 2 negative for dysplasia; and 1 inflamed gastric cardia without Barrett's. The thirteen trial phase endoscopy sets included: 2 adenocarcinomas; 8 high-grade dysplasias; 0 low-grade dysplasia; 1 indefinite for dysplasia; 1 negative for dysplasia; and 1 inflamed gastric cardia without Barrett's.

\section{Inter-Observer Agreement per Biopsy}

Table 2 indicates the percent agreement for each diagnosis on a per biopsy basis. Percent agreement 


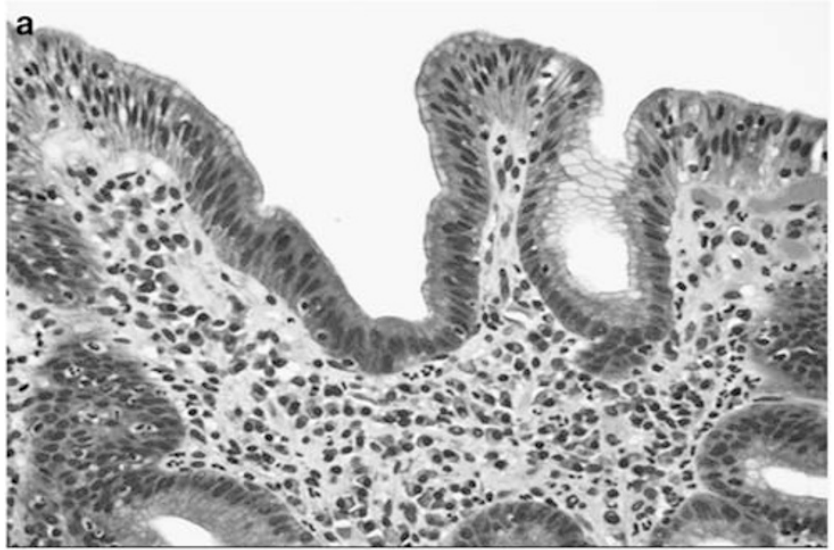

b

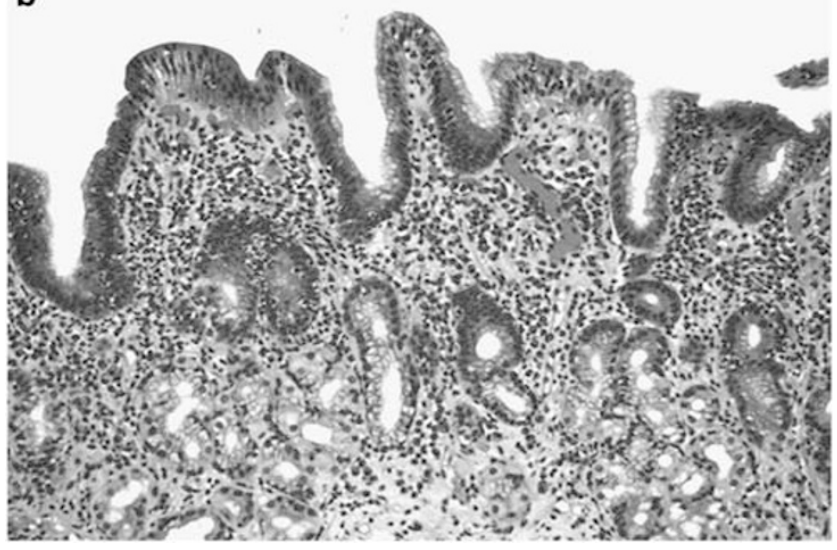

Figure 4 Inflamed and injured gastric mucosa in reflux disease (a) often mimics Barrett's dysplasia, because of consistent mucin loss, nuclear stratification, hyperchromasia, and atypia in both situations. Helpful features for identifying reactive cardia include preservation of a linear row of surface foveolar cell mucin caps (a), and the lower magnification features of villiform architecture and a so-called 'top-heavy' atypia. The latter refers to atypia limited to the upper half of the mucosa (b), in comparison to Barrett's mucosa that shows predominantly 'bottom-heavy' atypia that is usually most pronounced in the lower half of the mucosa (see Figures 2, 3, and 5).

was $90 \%$ or greater for all diagnoses when averaged overall rater pairs and both rounds (Kappa value 0.77). Agreement on all diagnoses except the presence of Barrett's metaplasia without dysplasia was higher in round 2 than round 1.

Univariate analysis for various factors affecting diagnostic agreement revealed that for highgrade dysplasia the factor with the largest absolute impact was pathologist assignment of uncertainty by diagnosing 'high-grade dysplasia cannot be excluded'. When this notation was present, the percent agreement on a diagnosis of high-grade dysplasia was $78 \%$ compared with $91 \%$ when this factor was absent $(P=0.06)$. Other potentially obscuring variables by univariate analysis were as follows: active inflammation $(86 \%$ agreement if present, $91 \%$ if absent, $P$-value 0.40 ); post- versus
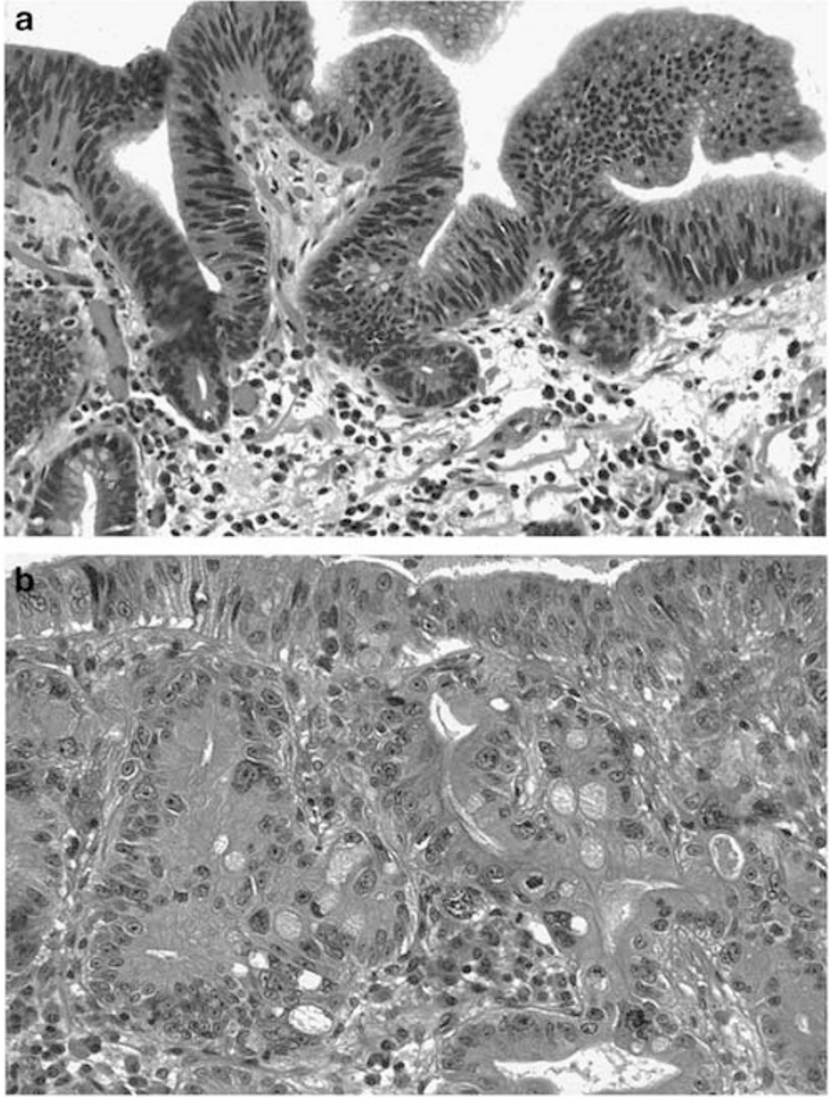

Figure 5 The most important criterion for distinguishing Barrett's low-grade dysplasia (a) from high-grade dysplasia (b) involves nuclear polarity. In low-grade dysplasia nuclear polarity is maintained, whereby the long axis of the nuclei remains perpendicular to the basal lamina and the nuclei remain orderly relative to each other (a). High-grade dysplasia classically exhibits loss of nuclear polarity, whereby the nuclei lose their pencillate shape, become disorganized and no longer maintain a perpendicular relationship to the basal lamina (b). Maintenance or loss of polarity is the most objective criterion available for the grading of intestinal-type dysplasia, but of course the more continuous variables of increasingly abnormal glandular architecture, and other cytological criteria, such as increasing nuclear size and pleomorphism, remain important and are all exhibited in these examples as well.

pre-endoscopic ablation therapy (92\% agreement versus $89 \%, P$-value 0.56 ); and the number of biopsies reviewed $(90 \%$ agreement if less than 16 biopsies, $90 \%$ if greater than 16 biopsies, $P$-value 0.97).

After adjustment for all planned covariates, the factor with the greatest impact on pathologist agreement and statistical significance was again assignment of uncertainty through the diagnosis of 'high-grade dysplasia cannot be excluded' (RR 2.9 [95\% CI $(1.3,6.5)] ; P=0.009)$. Other potentially obscuring variables by multivariate analysis revealed the following: active inflammation (RR 0.8 [95\% CI $(0.4,1.8)] ; P=0.61)$; post-endoscopic ablation therapy (RR 0.8 [95\% CI $(0.3,2.4)] ; P=0.74)$; and the number of biopsies reviewed (RR 1.1 [95\% CI $(0.3,3.6)] ; P=0.86)$. 
Table 2 Percent inter-observer diagnostic agreement by the three study pathologists on a per biopsy basis.

\begin{tabular}{lcccc}
\hline \multicolumn{5}{c}{$\begin{array}{c}\text { Three-way pathologist diagnostic percent agreement } \\
\text { per biopsy }\end{array}$} \\
\cline { 1 - 3 } & $\begin{array}{c}\text { Round \# 1 } \\
\text { 3-way \% } \\
\text { agreement }\end{array}$ & $\begin{array}{c}\text { Round \# } 2 \\
\text { 3-way \% } \\
\text { agreement }\end{array}$ & Average & $(95 \%$ CI) \\
Diagnosis & 88 & 92 & 90 & $(84,94)$ \\
\hline $\begin{array}{l}\text { High-grade dysplasia } \\
\text { Adenocarcinoma }\end{array}$ & 98 & 99 & 99 & $(94,100)$ \\
$\begin{array}{l}\text { High-grade dysplasia } \\
\text { or adenocarcinoma }\end{array}$ & 90 & 93 & 91 & $(85,94)$ \\
$\begin{array}{l}\text { Dysplasia } \\
\text { Barrett's }\end{array}$ & 87 & 93 & 90 & $(85,93)$ \\
\cline { 1 - 3 } & 96 & 92 & 94 & $(86,97)$ \\
\hline
\end{tabular}

Abbreviation: CI, confidence interval.

\section{Intra-Observer Agreement- Per Biopsy}

Intra-observer agreement per biopsy was very high for all outcomes. When averaged overall three pathologists, intra-observer agreement on the outcomes of high-grade dysplasia, adenocarcinoma, high-grade dysplasia or adenocarcinoma, dysplasia, and Barrett's esophagus without dysplasia was $93 \%$, 99\%, 94\%, 91\%, and 95\%, respectively. In multiple regression analysis, 'high-grade dysplasia cannot be excluded' had a significant impact on agreement for all outcomes after adjusting for other factors (data not shown).

\section{Discussion}

Prior to this study, the magnitude of the problem of overdiagnosing Barrett's high-grade dysplasia was essentially unknown. These data document that it is alarmingly high. Of the 485 international, multiinstitutional patients seeking nonsurgical ablation therapy for Barrett's high-grade dysplasia, a total of $194(40 \%)$ had either low grade or indefinite for dysplasia $(n=140)$, no dysplasia $(n=35)$, other $(n=1)$, or even lacked Barrett's altogether with only reflux disease $(n=18)$ upon reinterpretation by the study pathologists. Considering the potential consequence of unnecessary esophagectomy for these patients, these data are concerning.

The marked overlap between inflammatory, regenerative, metaplastic, and neoplastic change in Barrett's esophagus makes this a very-challenging diagnostic area. This study seeks to improve awareness among pathologists and clinicians on the extent of the problem and to help pathologists avoid the many pitfalls mimicking high-grade dysplasia in Barrett's esophagus.

The principle confounding histological factors in this series were one or more of: inflammatory change in Barrett's epithelium $(n=182)$; basal metaplastic glandular atypia with surface maturation $(n=147)$; lack of precise criteria to separate lower grades of neoplasia $(n=102)$; tangential sectioning artifact in Barrett's mucosa $(n=59)$; and reactive gastric cardia without Barrett's $(n=38)$.

Inflammatory atypia was the number one confounder in this series. With pronounced inflammation and particularly erosion/ulceration, the best criterion to exclude dysplasia is maturation of atypia as the epithelium extends onto the mucosal surface. ${ }^{5-8,11-15}$ Surface maturation, however, may be lacking altogether in marked inflammatory injury. The cytological features of inflammatory change can be indistinguishable from those of high-grade dysplasia. ${ }^{5-8,11-15}$ Caution should be exercised in the setting of prominent inflammation by diagnosing indefinite changes for dysplasia. ${ }^{5-8,11-15}$ If concern for high-grade dysplasia exists, a cautionary disclaimer indicating that high-grade dysplasia cannot be excluded is appropriate. This exact diagnosis achieved univariate and multivariate significance for predicting disagreement among the study pathologists, with a relative risk of 2.9 (95\% confidence interval 1.3, $6.5 ; P=0.009$ ), emphasizing the magnitude of the difficulty imposed by inflammatory change. If markedly inflamed biopsies are received, diagnostic caution by the pathologist is essential, along with the request for more biopsies following intensive medical therapy to attempt reduction of obscuring inflammatory change.

Another diagnostic pitfall is atypia limited to basal metaplastic glands nearest the muscularis mucosae. Barrett's mucosa is metaplastic and therefore more atypical at its nondysplastic baseline than normal intestinal mucosa. This metaplastic atypia ranges from mild to even occasionally marked, and is most pronounced at the basal or regenerative aspect of metaplastic mucosa. ${ }^{5-8,11,12}$ Surface maturation of basal atypia, in which the nuclei become smaller, normochromatic, uniform, and nonstratified as the epithelium extends onto the biopsy surface, is again the most important criterion available for excluding dysplasia, similar to its importance in inflammatory change. ${ }^{5-8,11-15}$

The proposed concept of crypt-limited dysplasia contradicts that surface maturation excludes dysplasia. ${ }^{13-15}$ Only 15 such crypt-limited cases have been reported in the literature in relation to the patients' corresponding Barrett's segments. ${ }^{14}$ These cases derived from a high-risk cohort of 208 Barrett's patients with a $61 \%$ prevalence of conventional dysplasia and cancer and are thus not representative of the general Barrett's population. They instead make up only a fraction of a very-high-risk Barrett's neoplasia cohort. Extrapolation of so-called crypt dysplasia to general practice is therefore questionable. Thirteen $(85 \%)$ of these rare cases of cryptlimited dysplasia also had conventional dysplasia or adenocarcinoma elsewhere in their Barrett's segments ${ }^{14}$, further obscuring the independent significance of this change as an isolated finding. Longitudinal data on crypt-limited dysplasia as an isolated finding are unavailable. A single observer 
variability study of 15 cases of crypt-limited dysplasia did not assess how it should be differentiated from inflammatory or regenerative change. ${ }^{14}$ Finally, an image analysis DNA ploidy study reported similar low-level ploidy alterations in nondysplastic Barrett's and crypt-limited dysplasia. ${ }^{15}$ Thus, the composite published data on crypt-limited dysplasia, all from the same group of investigators, do not support its use in diagnostic practice. Basal metaplastic glandular atypia, on the other hand, is a highly commonplace finding in most Barrett's mucosa that should not be over diagnosed as dysplasia, particularly high-grade dysplasia given its increased clinical significance. It cannot be overemphasized that surface maturation remains the mainstay criterion for excluding dysplasia in Barrett's mucosa, whether in inflammatory change or basal metaplastic glandular atypia.

Reactive change mimicking Barrett's dysplasia in inflamed gastric cardiac mucosa is frequent due to gastroesophageal reflux disease. Reactive cardia and dysplastic Barrett's share two prominent features, namely mucin loss and nuclear atypia, and as such can appear quite similar. The surface versus deep location of the most pronounced atypia can be helpful, but requires well-oriented and therefore large biopsies. Barrett's mucosa tends to have more 'bottom-heavy' atypia that is most pronounced nearest the muscularis mucosae. Alternatively, reactive gastric cardiac mucosa tends to have a 'top-heavy' distribution of atypia that is most pronounced in the surface half of the biopsy. These two zonal differences likely reflect different locations of the regenerative or stem cell compartments of intestinal versus gastric mucosal types. Specifically, the atypia in Barrett's mucosa is 'bottomheavy' because the regenerative zone resides at the base of Barrett's mucosa. Alternatively, the atypia in reactive gastric mucosa is 'top heavy' because the regenerative zone of gastric mucosa is in the mid or neck region.

Retention of linear, contiguous arrays of often attenuated foveolar cell mucin caps is another helpful feature supporting inflamed gastric cardiac mucosa over neoplastic Barrett's. While foveolartype gastric mucinous cells are variably admixed in Barrett's metaplastic epithelium, they are typically interspersed among scattered goblet cells rather than appearing as long contiguous arrangements along the surface or replacing complete glands, as they do in gastric mucosa.

Reliable distinction between indefinite to lowgrade dysplasia versus high-grade dysplasia is another essential element to avoiding the overdiagnosis of high-grade dysplasia. ${ }^{5-8,11-15}$ This factor contributed to 102 of the over diagnosed cases in this study. Maintenance of nuclear polarity in low-grade dysplasia is the most reliable criterion for this distinction, whereby the long axes of the nuclei remain parallel to one another and perpendicular to the basal lamina. High-grade dysplasia characteristically shows loss of polarity, with more irregular and disordered nuclei that lack a perpendicular orientation to the basal lamina. High-grade dysplasia often also exhibits greater degrees of nuclear enlargement, pleomorphism, stratification, and hyperchromasia, along with a greater distortion of glandular architecture than low-grade dysplasia. These latter criteria, however, are all continuous variables without precisely defined cutoffs and therefore more subjective. Loss of nuclear polarity is a more dichotomous criterion and therefore more objective for the separation of low-grade and highgrade dysplasia.

Tangential sectioning artifact, contributing to 59 cases over diagnosed with high-grade dysplasia in this study, can simulate nuclear stratification as a feature of dysplasia. A good clue to this artifact is that the cytoplasm will also be elongated with tangential sectioning. Further, nuclear stratification should probably not be used as a sole feature for a diagnosis of dysplasia. If the nuclei remain small and uniform and lack all other features of dysplasia, they are very likely not dysplastic. Tangential sectioning can also exaggerate basal glandular atypia if a horizontal section cuts across the basal compartment and obscures surface maturation. If the surface mucosa is not visualized in horizontally sectioned biopsies, additional levels may be helpful. If still unclear, a diagnosis of indefinite for dysplasia is probably more appropriate to avoid overdiagnosing a horizontal section through nondysplastic basal glands simulating dysplasia. ${ }^{5-8,11-15}$

Because of its recent description, the gastric variant of Barrett's dysplasia was not separately analyzed in this study. ${ }^{16-18}$ This omission is unlikely to have affected the reported results; however, because gastric-type Barrett's dysplasia represents a minority of dysplasia cases $(\sim 15 \%)$. It is also substantially more subtle than intestinal-type dysplasia, leading to under rather than over diagnosis, the subject of this study. Another shortcoming of this study is the lack of longitudinal follow-up on the 194 patients reinterpreted in this study to lack highgrade dysplasia. Benign outcome data for these patients would have added further support to the absence of high-grade dysplasia. The exclusion of these patients from the ablation trial forming the basis of the study precluded follow-up data.

The pathologists in this study achieved the highest reported diagnostic reproducibility in the literature for gastrointestinal glandular dysplasia. This and the rigorous design of the reproducibility study help to validate the presented findings. Overall three-way inter-observer percent agreement was $90 \%$ or greater for high-grade dysplasia when averaged overall three pathologists on a per biopsy basis (Kappa value 0.77). Inter-observer agreement in four earlier studies of Barrett's high-grade dysplasia range from $61 \%-83 \% .^{5,13,19,20}$ The improved agreement in the current study very likely reflects that all of the study pathologists were from the same 
practice group and closely aligned in their diagnostic approaches and criteria. The high level of reproducibility nonetheless helps support the validity of the reported diagnoses.

\section{Conclusions}

The over interpretation of high-grade dysplasia in Barrett's esophagus was highly prevalent in this study, involving 194 patients or $40 \%$ of the 485 total patient cohort. The most common diagnostic pitfalls are detailed, including inflammatory change, basal metaplastic atypia, imprecise criteria for lower grade neoplasia, tangential sectioning artifact, and inflamed cardia in reflux disease without Barrett's esophagus. Increased awareness of the prevalence and causes of this issue will hopefully improve diagnostic accuracy.

\section{Acknowledgments}

This work is dedicated to the cherished memory of the late Dr Rodger C Haggitt, without whose esteemed contributions as one of the great gastrointestinal pathologists of our time, and as a contributing pathologist to the study, it would not have been possible.

\section{Disclosure/conflict of interest}

The authors declare no conflict(s) of interest.

\section{References}

1 Barrett NR. The lower esophagus lined by columnar epithelium. Surgery 1957;41:881-894.

2 Wang KK, Sampliner RE. Updated guidelines 2008 for the diagnosis, surveillance and therapy of Barrett's esophagus. Am J Gastroenterol 2008 103:788-797.

3 Overholt BF, Lightdale CJ, Wang KK, et al. Photodynamic therapy with porfimer sodium for ablation of high-grade dysplasia in Barrett's esophagus: international, partially blinded, randomized phase III trial. Gastrointest Endosc 2005;62:488-498.

4 Overholt BF, Wang KK, Burdick JS, et al. Five-year efficacy and safety of photodynamic therapy with Photofrin in Barrett's high-grade dysplasia. Gastrointest Endosc 2007;66:460-468.
5 Montgomery E, Bronner MP, Goldblum JR, et al. Reproducibility of the diagnosis of dysplasia in Barrett esophagus: a reaffirmation. Hum Pathol 2001;32: 368-378.

6 Haggitt RC. Barrett's esophagus, dysplasia, and adenocarcinoma. Hum Pathol 1994;25:982-993.

7 Patil DT, Goldblum JR, Rybicki L, et al. Prediction of adenocarcinoma in esophagectomy specimens based upon analysis of preresection biopsies of barrett esophagus with at least high-grade dysplasia: a comparison of 2 systems. Am J Surg Pathol 2012;36:134-141.

8 Zhu W, Appelman HD, Greenson JK, et al. A histologically defined subset of high-grade dysplasia in Barrett mucosa is predictive of associated carcinoma. Am J Clin Pathol 2009;132:94-100.

9 Liang KY, Zeger SL. Longitudinal data analysis using generalized linear models. Biometrika 1986;73:13-22.

10 Cohen JA. A coefficient of agreement for nominal scales. Educ Psychol Assess 1960;2037-2046.

11 Downs-Kelly E, Mendelin JE, Bennett AE, et al. Poor interobserver agreement in the distinction of high-grade dysplasia and adenocarcinoma in pretreatment Barrett's esophagus biopsies. Am J Gastroenterol 2008;103:2333-2340.

12 Odze RD. Diagnosis and grading of dysplasia in Barrett's oesophagus. J Clin Pathol 2006;59:1029-1038.

13 Coco DP, Goldblum JR, Hornick JL, et al. Interobserver variability in the diagnosis of crypt dysplasia in Barrett esophagus. Am J Surg Pathol 2011;35:45-54.

14 Lomo LC, Blount PL, Sanchez CA, et al. Crypt dysplasia with surface maturation: a clinical, pathologic, and molecular study of a Barrett's esophagus cohort. Am J Surg Pathol 2006;30:423-435.

15 Zhang X, Huang Q, Goyal RK, Odze RD. DNA ploidy abnormalities in basal and superficial regions of the crypts in Barrett's esophagus and associated neoplastic lesions. Am J Surg Pathol 2008;32:1327-1335.

16 Rucker-Schmidt RL, Sanchez CA, Blount PL, et al. Nonadenomatous dysplasia in Barrett esophagus: a clinical, pathologic, and DNA content flow cytometric study. Am J Surg Pathol 2009;33:886-893.

17 Mahajan D, Bennett AE, Liu X, Bena J, Bronner MP. Grading of gastric foveolar-type dysplasia in Barrett's esophagus. Mod Pathol 2010;23:1-11.

18 Brown IS, Whiteman DC, Lauwers GY. Foveolar type dysplasia in Barrett esophagus. Mod Pathol 2010;23: 834-843.

19 Reid BJ, Haggitt RC, Rubin CE, et al. Observer variation in the diagnosis of dysplasia in Barrett's esophagus. Hum Pathol 1988;19:166-178.

20 Sonwalkar SA, Rotimi O, Scott N, et al. A study of indefinite for dysplasia in Barrett's oesophagus: reproducibility of diagnosis, clinical outcomes and predicting progression with AMACR (alpha-methylacyl-CoA-racemase). Histopathology 2010;56:900-907. 